\title{
Fluorescence Correlation Spectroscopy (FCS) module for advanced undergraduate laboratories
}

\author{
Nancy R. Forde, ${ }^{1, *}$ David Lee, ${ }^{1}$ and John Bechhoefer ${ }^{1}$ \\ ${ }^{1}$ Department of Physics, Simon Fraser University, 8888 University Drive, Burnaby, BC, Canada V5A 1S6
}

\begin{abstract}
Fluorescence correlation spectroscopy (FCS) is a technique used to characterize diffusion in biological and soft-matter systems. We have built and incorporated an FCS set-up into our junior physics lab and senior biophysics lab courses. Here, we provide information about its construction and about experimental modules performed by students in the lab.
\end{abstract}

\section{INTRODUCTION}

Many undergraduate physics curricula do not include the option of a strong focus on soft matter and biophysics, particularly in their laboratory courses. Fluorescence correlation spectroscopy (FCS) is a technique that is used by the biophysics and soft matter research communities for investigating and exploiting diffusion to learn about interacting molecular systems [1-4]. Based on experimental and analytical physics principles, FCS offers an excellent entry into the stochastic and quantitative world of biophysics, without the need to understand advanced biological concepts.

Fluorescence correlation spectroscopy analyses the temporal fluctuations in fluorescence from a sample to provide insight into its dynamics. To do so, a dilute solution of fluorescently labelled particles ('fluorophores') is illuminated by a tightly focused laser beam (Fig. 1a). When a fluorophore passes through the beam, it can absorb the light and emit fluorescent photons of a longer wavelength. Confocal detection of the fluorescence emission with a time-sensitive detector results in a detected signal only when a fluorophore is present in the focal volume. Time-dependent autocorrelation analysis of the detected signal then provides information on the average residency time in the focal volume, which depends on the size of the focal volume and on the diffusivity of the fluorescent object. Applications include anomolous diffusion in soft matter systems, DNA-binding interactions, selfassembly, and characterizing intracellular mechanics [1-4].

Our FCS module was designed for a senior undergraduate lab course in Biophysics, and has since been incorporated into a junior-level Physics lab course. Its aim is to introduce students to the concepts of fluorescence correlation spectroscopy, through experiments and data analysis. The instrument design is based upon one in the literature [5], but incorporates a newer way of computing correlations that works directly with single-photon counts, rather than intensities [6]. In this report, we describe the experimental set-up (given prebuilt to the students), the analytical methods used by the students to interpret their data, and some examples of experimental modules designed to introduce students to different aspects of FCS.

*nforde@sfu.ca

\section{METHODS}

\section{A. Instrumentation}

A schematic of the FCS instrument is shown in Fig. 1b, with a description of the components provided in the caption. The light path is in one plane, which facilitates alignment, pedagogical understanding and safety. Briefly, light from a $532 \mathrm{~nm}$ laser pointer $(5 \mathrm{~mW})$ is used as the illumination source; it is focused by a high-numerical-aperture (NA) objective lens into a sample chamber. Confocal detection of the fluorescence emission from fluorophores in the sample chamber is achieved by reimaging the focal volume onto the pinhole-like aperture at the end of an optical fibre, which directs the light to a photomultiplier tube (PMT). Because standard fibres are not light-tight, we made a custom light-tight patchcord by starting with a conventional fibre stock and encasing it in black heat-shrink tubing. After shrinking the tubing, one puts on the fibre-coupling connectors. The PMT and associated data-acquisition system need to have high sensitivity and low background counts, and high temporal resolution. These requirements are necessary to detect low signal intensity from small numbers of fluorophores and to be sensitive to timescales significantly less than the typical passage time through the focal volume. We opted to use a PMT with endon detection for increased detection efficiency (Hamamatsu H10682-110), and our photon arrival times are recorded using a multi-function data acquisition card (National Instruments $6215 \mathrm{DAQ}$, using the $80 \mathrm{MHz}$ internal reference clock for counter measurements).

\section{B. Experimental Set-up}

To conduct an experiment, students must first load the sample of interest (see below for options) into a sample chamber. Sample chambers are constructed by sandwiching two pieces of double-sided sticky tape between a glass slide and a cover slip [7]. The cover slip thickness is dictated by the objective lens. Alternatively, students can melt thin strips of Parafilm to create the spacer gasket in the chamber. The width of channel through the chamber, between the spacers, is $\approx 5 \mathrm{~mm}$. For a 1" square cover slip, this results in a sample volume of $<20 \mu \mathrm{L}$. Samples are loaded using a micropipette, and the chambers are sealed at each end with either a drop of melted paraffin wax (preferred) or nailpolish. 
(a)

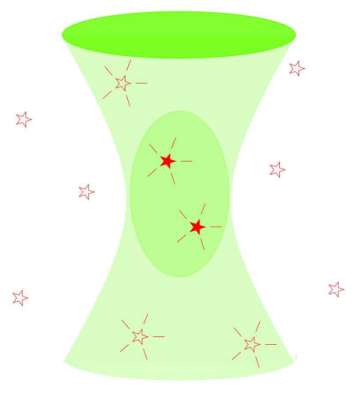

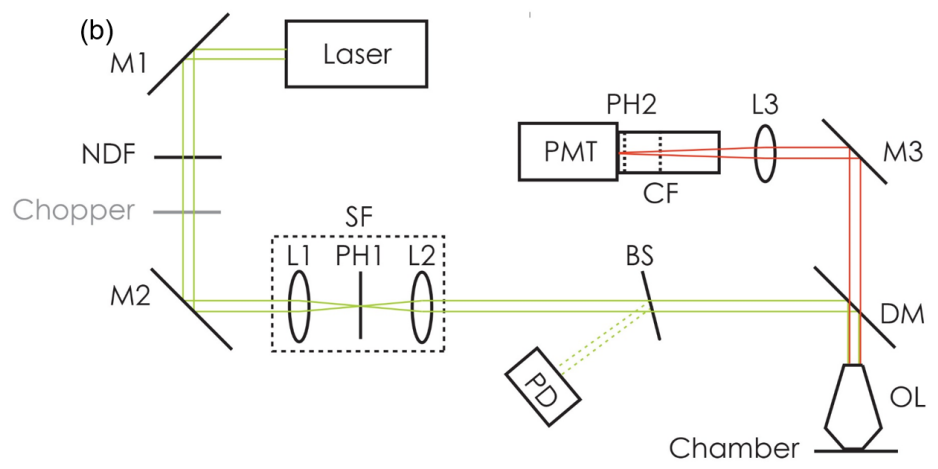

FIG. 1. (a) A dilute solution of fluorophores (stars) diffuses in solution. When one passes through a tightly focused laser beam (green), it can be excited and emit fluorescence (rays). Fluorescence light emanating from the laser focus (dark green ellipsoid; filled stars) is acquired via confocal detection and is autocorrelated to produce the FCS signal. (b) Experimental set-up. The green laser beam (532 nm, $5 \mathrm{~mW}$ diode laser) is expanded by lenses L1 and L2 and steered by three mirrors (M1, M2 and DM) into the objective lens OL, which focuses the light in the sample chamber. A neutral-density filter (NDF) attenuates the light so that only $\approx 100 \mu \mathrm{W}$ illuminates the sample. In the introductory module, a chopper wheel is introduced to spatially modulate the light intensity. The spatial filter SF containing a pinhole PH1 is used to 'clean up' the laser beam, passing a TEM00 mode to the chamber. The beam splitter (BS; glass coverslip) picks off a small fraction of the light and directs it to a photodiode (PD), which is used to track any variations in laser intensity. The objective used in these experiments is a water-immersion lens with a numerical aperture of 1.23 . The sample chamber is mounted to a one-axis translation stage (not shown), which allows for correct positioning of the chamber along the optic axis so that the laser focuses in the sample chamber. Emitted orange/red fluorescence passes through the dichroic mirror DM, and is steered by mirror M3 towards the photomultiplier tube PMT. Lens L3 focuses the fluorescent light, and the color filter CF blocks any stray/transmitted $532 \mathrm{~nm}$ light. Our set-up uses for pinhole PH 2 the end of an optical fibre that directs the light to the PMT; PH2 is positioned along the optic axis to allow light emitted only from within the focal volume to pass to the PMT. The PMT sends a pulse each time a photon hits it; the arrival times of these pulses are read by the data acquisition card on the computer.

Once prepared, the sample chamber is manually positioned on the single-axis translation stage, with the slide held in place by two rare-earth-magnets. The stage is moved carefully towards the water-immersion objective lens, and, when close enough, a drop of water is added between the lens and the chamber. The stage is then moved closer to the lens so that the laser focus falls within the sample chamber. If the chamber is filled with a sufficiently high concentration of fluorophore, an orange spot of light can be seen on an index card held between DM and M3; the orange light is fluorescence emission from the sample. After the sample chamber is correctly positioned, the neutral-density filter is re-inserted into the beam path, and only then is the PMT turned on. This approach minimizes the potential for damage to the PMT.

To align the signal on the PMT, students adjust the location of $\mathrm{PH} 2$, which is mounted on a three-axis positioner, in order to maximize the number of counts recorded on the PMT. It is useful for them to observe that the counts fall off much more rapidly when the pinhole is translated in $\mathrm{x}$ and $\mathrm{y}$, orthogonal to the beam path, than along the optical axis. This maps the elongated ellipsoidal shape of the focus in the sample chamber.

\section{Analytical methods}

In our implementation, the fluorescence intensity autocorrelation function $G(\tau)$ is determined directly from photon arrival time intervals rather than from explicit autocorrelation of the time-dependent intensity. Thus, we avoid the need for a hardware correlator such as in Ref. [8]. We adapted an existing algorithm for this purpose [6], and use a $\mathrm{C}++$ code called within the main LabVIEW interface to calculate $G(\tau)$.

The autocorrelation function for particles with a given diffusion constant $D$ is given by

$$
G(\tau)=\frac{1}{V_{\text {eff }}\langle C\rangle} \frac{1}{\left(1+\frac{\tau}{\tau_{\mathrm{D}}}\right)} \frac{1}{\sqrt{1+\frac{\tau}{\tau_{\mathrm{D}}}\left(\frac{r_{0}}{z_{0}}\right)^{2}}} .
$$

Equation 1 assumes the particles diffuse through an elliptical focal volume of radius $r_{0}$ and length along the optical axis $z_{0}$. See Ref. [9] for an outline of the derivation. In Eq. 1, $V_{\text {eff }}$ is the effective focal volume $\left(V_{\text {eff }} \approx \pi^{\frac{3}{2}} r_{0}^{2} z_{0}\right),\langle C\rangle$ is the average particle concentration in solution, and $\tau_{\mathrm{D}}$ is the correlation time for translational diffusion across the isotropic part of the ellipsoid. This characteristic time $\tau_{\mathrm{D}}$ is given by

$$
\tau_{\mathrm{D}}=\frac{r_{0}^{2}}{4 D}
$$

The expression $\tau_{\mathrm{D}}=\frac{r_{0}^{2}}{4 D}$ is characteristic of two-dimensional diffusion; it is used in Eq. 1, since the radius $r_{0}$ of the focal 
volume is so much narrower than its length $z_{0}$ and dictates the dominant timescale of fluctuations. In the limit $r_{0} \ll z_{0}$, Eq. 1 simplifies to

$$
G(\tau)=\frac{1}{V_{\mathrm{eff}}\langle C\rangle} \frac{1}{\left(1+\frac{\tau}{\tau_{\mathrm{D}}}\right)} .
$$

We find this form to give more reliable fitting results.

The size of the effective focal volume can be determined experimentally using fluorescent particles of known diffusion constant $D$. The expected value of $D$ for spheres of radius $R$ diffusing freely in a solution of viscosity $\eta$ at temperature $T$ can be calculated using the Stokes-Einstein relation,

$$
D=\frac{k_{\mathrm{B}} T}{\gamma}=\frac{k_{\mathrm{B}} T}{6 \pi \eta R},
$$

where $k_{\mathrm{B}}$ is Boltzmann's constant.

\section{EXPERIMENTAL MODULES}

\section{A. Introduction to autocorrelation}

To introduce students to the concept of autocorrelation, they are assigned the task of sketching the temporal correlation between one period of a square wave pulse and an infinite square wave of the same periodicity. The formal definition of the intensity autocorrelation function is useful for this exercise:

$$
G(\tau)=\frac{\langle\delta I(t) \delta I(t+\tau)\rangle}{\langle I(t)\rangle^{2}}
$$

Here, $\delta I(t)$ represents the difference in fluorescence intensity at time $t$ from its average value $\langle I(t)\rangle$, and can be positive or negative. $\tau$ indicates the time lag at which the correlation is calculated. The students should obtain a sawtooth form for $G(\tau)$ and are asked to plot this with time on a logarithmic axis, as this is the display format generally used for FCS.

Following their prediction, the students insert a chopper wheel into the beampath (Fig. 1) and a high-concentration solution of rhodamine $6 \mathrm{G}$ fluorophore $(1-10 \mu \mathrm{M})$ into a sample chamber. Students record autocorrelation curves of the temporally modulated excitation laser beam and are asked to determine how their appearance depends on the chopping rate.

\section{B. Focal volume determination}

The main focus of the experimental module is the determination of the diffusion coefficient of unknown particles (see following subsection). To do so, students must first determine the size of the focal volume using a sample of known diffusion constant. We have found a dilute solution of 100nm-diameter fluorescent beads (F8800 FluoSpheres, ThermoFisher) to work well for this purpose, as the large num- ber of fluorophores on each particle generates a bright emission signal as it diffuses through the focal volume. An autocorrelation curve is obtained, such as shown in Fig. 2. Students fit this measured autocorrelation curve with Eq. 3 to obtain the average number of particles in the focal volume, $\langle N\rangle=V_{\text {eff }}\langle C\rangle$, and the characteristic correlation time $\tau_{D}$. For the 100-nm-diameter spheres used here, $D=4.4 \times 10^{13}$ $\mathrm{m}^{2} \mathrm{~s}^{-1}$ from Eq. 4, which is used to determine $r_{0}$ using Eq. 2. The measurements of Fig. 2 give $r_{0}=460 \mathrm{~nm}$, slightly larger than the diffraction limit for $\lambda=532 \mathrm{~nm}$, as expected. Students can also observe an increase in $G(0)$ when they measure a bead solution of lower concentration.

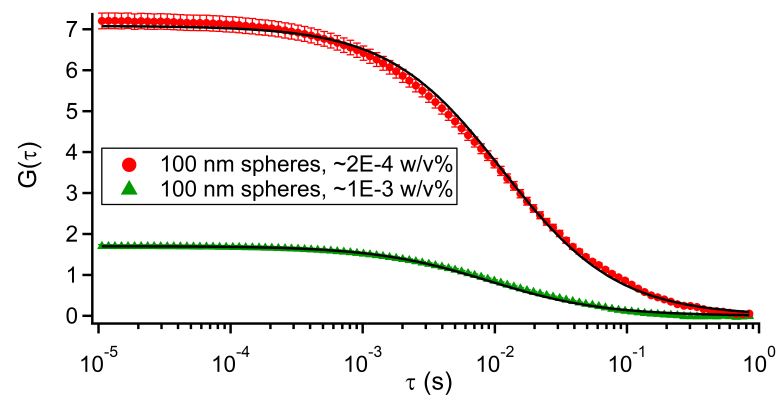

FIG. 2. Autocorrelation curves obtained for $100 \mathrm{~nm}$ spheres at two different concentrations (weight/volume), fit with Eq. 1. Both exhibit the same characteristic time $\tau_{\mathrm{D}} \approx 12 \mathrm{~ms}$; the plateau is higher for the lower-concentration sample because of its lower $\langle N\rangle$.

\section{Determination of diffusion coefficients}

\section{Beads of unknown size}

Students are given a solution of fluorescent beads of 'unknown' size. They measure an autocorrelation curve and determine $\tau_{\mathrm{D}}$. Using their precalibrated focal volume size, they determine the size of their particles using eqs. 2 and 4. As an extension, they can predict and then measure $G(\tau)$ for a mixture of particles of two different sizes.

\section{Fluorescent dye}

The fluorescent dye rhodamine 6G (R6G, used in the chopper wheel experiments) is much smaller and fainter than the fluorescent beads used in the experiments. As such, it diffuses more rapidly through the focal volume and gives rise to smaller amplitude intensity fluctuations. In our instrument, it is challenging to detect and fit $G(\tau)$ from R6G in water; however, slowing its diffusion down by adding glycerol to the solution generates clear curves. The relationship between diffusion constant and viscosity can be determined by measuring solutions containing varying amounts of glycerol (Fig. 3). 
(a)
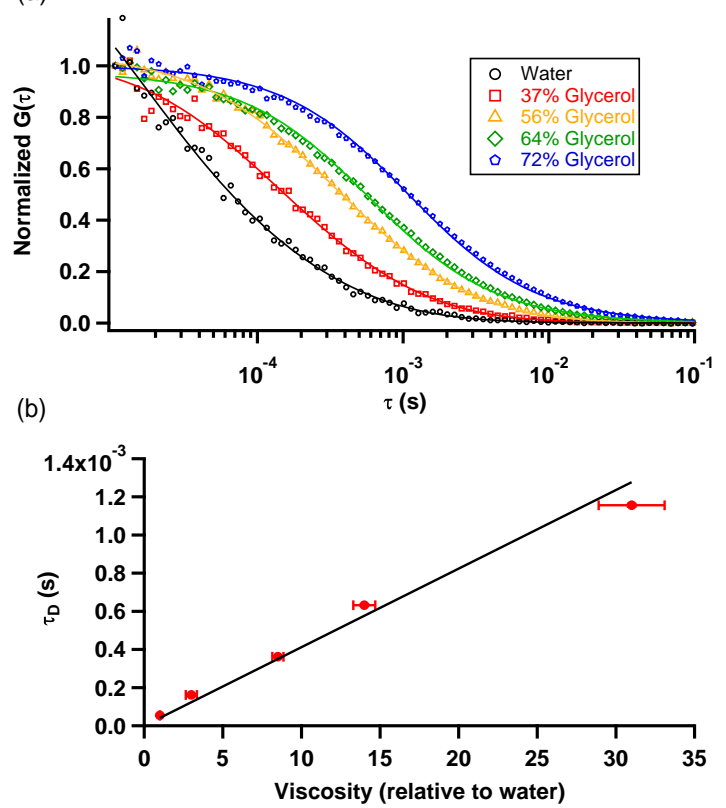

FIG. 3. Rhodamine $6 \mathrm{G}$ autocorrelation analysis. (a) As the viscosity of the solution increases (due to increasing glycerol content), diffusion of the fluorophore slows and the autocorrelation time increases. Fits of normalized $G(\tau)$ data use Eq. 1 to obtain $\tau_{\mathrm{D}}$. (b) $\tau_{\mathrm{D}}$ scales linearly with the viscosity of the solution, as expected.

\section{DNA}

In a more biologically related application, students measure the autocorrelation curve of fluorescently labelled DNA, and then determine how the size they obtain for DNA compares with predictions of polymer theory. This module requires more sample preparation and more physics knowledge than the others. It is also richer, as it can be extended in more directions. An alternative FCS experiment with DNA is provided in Ref. [5].

DNA can either be provided to the students or the students can prepare it themselves. In our biophysics laboratory course, students learn to prepare and linearize plasmid DNA in a separate module, and then use this DNA when they conduct their FCS experiments. Protocols for DNA preparation are available from the authors. For measurement, DNA is incubated in a TE buffer solution with SybrSafe dye; this dye is fluorescent when bound to double-stranded DNA and otherwise does not fluoresce. It is less toxic than other DNAbinding dyes such as ethidium bromide and is commonly used in undergraduate molecular biology labs for DNA electrophoresis experiments. In solution, polymers adopt a random coil geometry, which maximizes their configurational entropy. Thus, determining the correlation time $\tau_{\mathrm{D}}$ of this fluorescent DNA allows an estimate of its effective size in solution; this can be compared to the predictions of polymer theory for semiflexible polymers. Polymer theory predicts that the radius of gyration is given by $R_{g} \approx \sqrt{p L / 3}$ ) [10], where the persistence length $p=50 \mathrm{~nm}$ for double-stranded DNA in most salt solutions. While technically FCS measures the radius of hydration of solvated molecules, this is of the same order as radius of gyration and serves to introduce the concept here.

\section{CONCLUSIONS}

We have built and developed experimental modules to introduce advanced Physics students to FCS. The modules described here can be extended into more substantial independent projects. For example, the diffusion and size of DNA of different lengths and of different topologies could be characterized $[10,11]$. This could be complemented with experiments using other techniques: in our biophysics laboratory course, students separately investigate the mobility of these different topological forms of DNA in electrophoresis experiments. The literature holds suggestions for many other possible applications of FCS [1-4], many of which are compatible with the advanced undergraduate Physics laboratory.

\section{ACKNOWLEDGMENTS}

NRF thanks the Research Corporation for a Cottrell Scholar Award. We thank Thomas Luo for constructing the initial version of this instrument, Barbara Frisken for contributions to the manuscript, Art Bailey for technical assistance, and countless students for their feedback on the module.
[1] D.S. Banks, C. Fradin. Biophys. J. 89, 2960 (2005)

[2] E. Haustein, P. Schwille. Annu. Rev. Biophys. Biomol. Struct. 36, 151 (2007)

[3] K. Gurunathan, M. Levitus. Prog. Nucleic Acid Res. Mol. Biol. 82, 33 (2008)

[4] E.L. Elson. Biophys. J. 101, 2855 (2011)

[5] R. Rieger, C. Rocker, G.U. Nienhaus. Am. J. Phys. 73, 1129 (2005)

[6] T.A. Laurence, A.N. Kapanidis, X.X. Kong, D.S. Chemla, S. Weiss. J. Phys. Chem. B 108, 3051 (2004)
[7] D.C. Appleyard, K.Y. Vandermeulen, H. Lee, M.J. Lang. Am. J. Phys. 75, 5 (2007)

[8] B.D. Gamari et al. Am. J. Phys. 82, 712 (2014)

[9] P. Schwille, E. Haustein. Biophysics Textbook Online (2001)

[10] D.R. Latulippe, A.L. Zydney. Biotech Bioeng. 107, 134 (2010)

[11] R.M. Robertson, S. Laib, D.E. Smith. PNAS 103, 7310 (2006) 